Published in final edited form as:

Urol Oncol. 2017 April ; 35(4): 151.e9-151.e15. doi:10.1016/j.urolonc.2016.11.002.

\title{
Prognostic value of lymph node yield during nephroureterectomy for upper tract urothelial carcinoma
}

\author{
Andrew G. Winer ${ }^{1}$, Emily A. Vertosick ${ }^{2}$, Mazyar Ghanaat ${ }^{3}$, Renato B. Corradi ${ }^{3}$, Sigrid \\ Carlsson $^{2,4,5}$, Daniel D. Sjoberg ${ }^{2}$, Alexander I. Sankin ${ }^{6}$, John P. Sfakianos ${ }^{7}$, Eugene K. \\ Cha $^{3}$, Guido Dalbagni ${ }^{3}$, and Jonathan A. Coleman ${ }^{3}$ \\ ${ }^{1}$ SUNY Downstate College of Medicine and Kings County Hospital Center, New York, USA \\ ${ }^{2}$ Department of Epidemiology and Biostatistics, Memorial Sloan Kettering Cancer Center, New \\ York, USA \\ ${ }^{3}$ Urology Service at the Department of Surgery, Memorial Sloan Kettering Cancer Center, New \\ York, USA \\ ${ }^{4}$ Department of Surgery, Memorial Sloan Kettering Cancer Center, New York, USA \\ ${ }^{5}$ Sahlgrenska Academy at the University of Gothenburg, Gothenburg, Sweden \\ ${ }^{6}$ Department of Urology, Montefiore Medical Center and Albert Einstein College of Medicine, New \\ York, USA \\ ${ }^{7}$ Department of Urology, Icahn School of Medicine at Mount Sinai, New York, USA
}

\section{Abstract}

Introduction-Lymph node dissection (LND) performed during radical nephroureterectomy (RNU) for upper tract urothelial carcinoma (UTUC) remains controversial and difficult to evaluate. The aim of this study was to investigate whether removal of more lymph nodes during RNU is safe and improves oncologic outcomes.

Methods-We evaluated 422 patients who underwent RNU with concomitant LND for UTUC between 1976 and 2015, assessing for an association between total nodes removed, recurrence-free (RFS) and cancer-specific survival (CSS) using Cox proportional hazards models. We also investigated the relationship between nodal yield and peri-operative metrics and inter-surgeon variability using linear regression.

Results-In our cohort of 442 patients, 239 developed recurrences and 94 patients died from disease. Median follow-up among survivors was 3.7 years (IQR 1.2, 7.4). The median nodal yield was 9 (IQR 4, 16). Among patients with node positive disease (pN1), we observed a significant

Correspondence: Jonathan A. Coleman, Department of Surgery, Urology Service, Memorial Sloan Kettering Cancer Center, 1275 York Ave, New York, NY 10065, colemanj@ mskcc.org, Telephone: 646-422-4432, Fax: 212-452-3323.

Publisher's Disclaimer: This is a PDF file of an unedited manuscript that has been accepted for publication. As a service to our customers we are providing this early version of the manuscript. The manuscript will undergo copyediting, typesetting, and review of the resulting proof before it is published in its final citable form. Please note that during the production process errors may be discovered which could affect the content, and all legal disclaimers that apply to the journal pertain.

Disclosure of Potential Conflicts of Interest: None 
improvement in RFS (HR 0.84 per 5 nodes removed, $\mathrm{p}=0.039$ ) and a non-significant improvement in CSS with an increase in the nodal yield (HR 0.90 per 5 nodes removed, $\mathrm{p}=0.2$ ). There was no evidence of an association between node yield and OR time, EBL or 30 day complications on multivariable analysis. There was significant heterogeneity between surgeons regarding the extent of lymph node dissection $(\mathrm{p}<0.0001)$.

Conclusions-We found that a more extensive node dissection may improve oncologic outcomes in a subset of high-risk patients without significantly increasing OR time or serious complications. Additionally we identified considerable inter-surgeon heterogeneity with regards to the extent of lymph node dissection furthering the notion of surgeon variability as a nonstandardized factor.

\section{Keywords}

Upper tract urothelial carcinoma; UTUC; extent of lymph node dissection; oncologic outcomes; complications

\section{Introduction}

Lymph node (LN) metastases in upper tract urothelial carcinoma (UTUC) are present in up to $30 \%$ of high-risk patients and are an independent predictor of worse cancer-specific outcomes $^{1,2}$. Although radical nephroureterectomy (RNU) with bladder cuff excision is the standard treatment for $\mathrm{UTUC}^{3}$, the therapeutic role of concurrent lymph node dissection (LND) remains debated and its practice is not standardized ${ }^{4}$. The EAU guidelines recommend lymphadenectomy for invasive UTUC, although only with a grade $\mathrm{C}$, stating that the indication and extent of LND cannot be standardized ${ }^{5}$.

Regional LNs are the most common route for metastatic progression. Lymphadenectomy is therefore important for optimal disease staging and risk stratification for potential adjunctive therapies ${ }^{6,7}$, although neoadjuvant chemotherapy is preferred because of compromise in renal function after RNU. The survival benefit of LND is not clear with some studies showing support ${ }^{8-10}$, and some do not ${ }^{11,12}$, none of which are randomized prospective trials. Therefore, in patients with locally advanced UTUC, the potential therapeutic benefit of extended lymphadenectomy is controversial ${ }^{8,10,12-15}$. The primary aim of this study was to evaluate the association between LN yield during RNU for UTUC and recurrence-free (RFS) and cancer-specific survival (CSS). Secondarily, we investigated inter-surgeon variability with regards to extent of LND and its effect on peri-operative metrics such as OR time, blood loss and 30-day complications.

\section{Methods}

After obtaining institutional review board approval, we identified 642 patients who had undergone nephroureterectomy for UTUC at Memorial Sloan Kettering Cancer Center between 1976-2015. Among these 642 patients, 442 underwent LND and were included in our cohort. All 442 patients had a formal LND, which was left to the surgeon's discretion but generally included dissection of nodes surrounding the renal vessels and along the 
sidewall of the ipsilateral great vessel (aorta:right, cava:left). Multiple pathologists were involved, all highly trained and with expertise in the field.

Complications were recorded and graded based on a modified Clavien-Dindo classification system of surgical complications, and were retrospectively reviewed ${ }^{16}$. Linear regression was used to assess the association between the number of nodes removed and EBL and OR time. Logistic regression was used for complications within 30 days. To determine the association between total nodes removed and RFS and CSS, we used Cox proportional hazards models. For all outcomes, both univariate models, with nodal yield as a predictor, and multivariable models were used. Multivariable models were adjusted for the number of nodes as well as age, smoking history, tumor focality, receipt of preoperative chemotherapy, ASA score (I/II or III/IV), tumor grade (low or high) and pathologic T stage (lower than T2 vs T2 and higher). Tumor within the bladder after RNU was not considered as recurrence. Patients who developed muscle invasive bladder cancer were excluded.

We hypothesized that while the nodal yield would not be associated with survival among node positive patients, removing an increased number of nodes among node negative patients could result in improved outcomes due to the potential elimination of micrometastatic nodal disease. We created Cox regression models including an interaction term between nodal status (positive or negative) and nodal yield to assess whether nodal yield affected RFS or CSS differently by nodal status. As sensitivity analyses, we applied these models to patients with high-grade tumors only and to patients with high-grade tumors who did not have pre-operative chemotherapy. Since treatment practices change over time, an additional sensitivity analysis used Cox regression models to assess whether the effect of number of nodes removed on node positive compared to node negative patients differed before and after the introduction of laparoscopic surgery at our institution in 2003. Since the number of LNs removed during LND varies by surgeon and may be a reflection of the extent or aggressiveness of a patient's disease at the time of surgery, we also aimed to identify any specific presurgical characteristics associated with the number of LNs removed. We used Kruskal-Wallis tests to assess whether any of the following characteristics were associated with the extent of LND: smoking history, pathologic stage (CIS/Ta/T0/T1/T2/T3/T4), pathologic grade (low/high), tumor focality (unifocal/multifocal), ASA score (I/II or III/IV), presence of concomitant CIS, receipt of pre-operative chemotherapy, open or laparoscopic surgery, and surgeon.

Since some surgeons perform a relatively standard LND on all patients while others remove only nodes which seem suspicious on imaging or intraoperatively, we aimed to assess the variation in the number of nodes removed by surgeon. We combined patients treated by surgeons performing $<10$ cases into one group, while surgeons who performed $>10$ cases were considered separately. There were 7 surgeons who performed $>10$ cases (one surgeon each performed 17, 31, 37, 39, 61, 110, and 114 procedures) and 33 procedures performed by surgeons who did $<10$ procedures each. All analyses were conducted using Stata 13 (StataCorp., College Station, Tx). 


\section{Results}

Patient and disease characteristics for patients who underwent nephroureterectomy and LND are presented in Table 1. Median follow-up for survivors was 3.7 years (IQR 1.2, 7.4). There were 239 recurrences and 94 died from disease. Median time from date of surgery to recurrence was 1.8 years $(95 \% \mathrm{CI} 1.2,2.9)$ and the 5-year RFS for the entire cohort was $39 \%$ (95\% CI 33\%, 44\%). The distribution of the number of LNs removed is shown in Figure 1. The median number of LNs removed was 9 (IQR 4, 16); 78 (18\%) had positive nodes, with a median of 2 involved nodes in node positive cases (IQR 1, 5). The nodal yield for laparoscopic/robotic (MIS) procedures was higher (median 11, IQR 6, 18) than for open procedures (median $8, \mathrm{IQR} 3,15, \mathrm{p}=0.0005$ ). Patients treated with pre-operative chemotherapy had an increased number of nodes removed compared to patients who did not receive chemotherapy [median of 13 (IQR 6,18) vs 8 (IQR 3, 15) nodes, respectively $(\mathrm{p}=0.001)$, Table 2]. There was evidence that patients with high-grade tumors also had more nodes removed (median of 9 nodes, IQR 4, 16, $\mathrm{p}=0.051$ ) than patients with low grade disease (median of 7 nodes IQR 3, 12).

We also found significant heterogeneity between surgeons in nodal yield $(\mathrm{p}<0.0001$, Figure 2). For peri-operative outcomes, we found no evidence of an association between nodal yield and OR time or EBL on univariate or on multivariable analysis (Table 3). The number of nodes removed was associated with the odds of any complication within 30 days on univariate analysis (OR 1.18 per 5 nodes, $95 \%$ CI 1.05, 1.32, p=0.004) although there was no evidence of increased risk of a grade 3 or higher complication within 30 days (OR 1.04 per 5 nodes, $95 \%$ CI $0.85,1.28, \mathrm{p}=0.7)$.

In the cohort as a whole we found no evidence that the extent of LND influenced RFS or CSS on univariate or multivariable analysis (Table 4). However, for RFS, we found a significant interaction between the number of nodes removed and nodal status on multivariable analysis $(\mathrm{p}=0.044)$, suggesting the number of nodes removed is associated with RFS differently depending on a patient's nodal status. Among patients with negative nodes, there was no evidence of an association between nodal yield and RFS ( $\mathrm{p}=0.8$, Table 4) or CSS $(\mathrm{p}=0.6)$. The analysis among patients with positive nodes however showed a significant improvement in RFS ( $\mathrm{p}=0.039)$ and a non-significant increase in CSS $(\mathrm{p}=0.2)$ with an increase in nodal yield.

Several sensitivity analyses were performed. The analyses of RFS and CSS were repeated including 331 patients with high-grade tumors only and 254 patients with high grade tumors who did not undergo pre-operative chemotherapy. Consistent with the main analyses, there was no evidence that extent of LND was associated with RFS or CSS on univariate or multivariable analysis in either cohort (Table 5). Among only high-grade tumors, there was evidence of improved RFS with an increasing number of nodes removed in node-positive patients, but not in node-negative patients (interaction $\mathrm{p}=0.053$, Table 6 ). When excluding high grade patients who received pre-operative chemotherapy, there was no longer evidence of an interaction between number of nodes and nodal status for RFS ( $\mathrm{p}=0.13$ ), although effect sizes are similar (Table 6), suggesting that this is due to a decrease in sample size. There was no evidence that number of nodes removed influenced CSS differently by nodal 
status among all high grade patients tumors or among high grade tumors excluding preoperative chemotherapy ( $\mathrm{p}>0.9$ and $\mathrm{p}=0.8$, respectively).

We investigated whether there was a difference in the effect of total nodes removed on RFS or CSS based on when surgery was performed by testing for an interaction between total nodes removed and LND status separately for patients treated before and after 2003. Due to the number of events in each group, we were limited to univariate analyses. We found no evidence that the total number of nodes removed affected RFS differently based on nodal status in patients treated before $2003(\mathrm{p}=0.4)$ but did find evidence of an interaction among patients treated in 2003 or later $(\mathrm{p}=0.05)$. Among patients treated in 2003 or later, the number of nodes removed did not influence RFS among node-negative patients but removing more nodes resulted in better RFS among node-positive patients (Table 6). There was no evidence of an interaction between number of nodes removed and nodal status for CSS in patients treated before or after 2003 ( $\mathrm{p}=0.6$ for both).

\section{Discussion}

Using this large, single-institution dataset we attempt to add to the limited literature on the controversial subject of LND in surgical management of patients with UTUC, and found limited evidence that the extent of LND had an influence on RFS in pN1 patients, suggesting improved RFS with an increasing number of nodes removed in node-positive patients. Due to lack of sensitivity of preoperative $\mathrm{CT}$ scans to identify $\mathrm{pN} 1{ }^{17}$ this limits the clinical usefulness of our results since the extent of LND had oncological impact only in pN1 patients. Predefined standardized templates could be the best solution as proposed by Kondo et al in two studies ${ }^{9,18}$, further discussed below. We found no evidence that the number of nodes removed influenced CSS differently by nodal status.

Removal of more LNs did not significantly prolong operative time, was not associated with higher EBL or grade 3 or higher complications at 30 days suggesting that a more extensive LND can be safely and efficiently performed in addition to the RNU. These findings may be attributed to level of surgeon expertise and high volume tertiary care center, but are consistent with findings reported by Kondo et al of template-based LND did not significantly increase the incidence of all grade complications ${ }^{18}$.

Our findings demonstrating that higher nodal counts during RNU carries independent predictive value in $\mathrm{pN} 1$ patients is unique from previous studies. Roscigno et al initially reported in 95 patients with muscle invasive UTUC that the number of nodes removed during LND was an independent predictor of CSS in the overall cohort and in a sub-analysis of the pN0 patients alone ${ }^{10}$. However, in a corollary multi-institutional consortium study of 552 patients, the authors were unable to identify a significant association between number of LNs excised and patient outcomes in the overall cohort but found that higher nodal yield carried a protective effect in the $\mathrm{pN} 0$ cohort alone ${ }^{15}$. One proposed rationale for the benefit observed in this specific group relates to the concept of locoregional control of micrometastases. A recent investigation from Japan re-evaluated the resected LN specimens from 52 patients who were initially deemed $\mathrm{pN} 0$ with anti-cytokeratin immunohistochemistry to identify occult metastatic disease. The authors detected 
micrometastases in $14 \%$ of cases suggesting that therapeutic benefit from LND may lie in the elimination of such sites of early spread of disease ${ }^{11}$. Although we did not specifically identify a therapeutic signal in the pNO cohort of patients in our study, the more important link between these investigations may lie in the eradication of locoregional disease ultimately resulting in improved oncologic outcomes.

Currently preoperative staging of the regional LNs in patients with UTUC is limited in accuracy ${ }^{17}$, therefore studies have suggested that template directed LND may be more important than absolute number of nodes removed. Kondo et al evaluated 109 patients with clinically node negative disease who underwent RNU and LND. The authors were unable to identify a minimum number of nodes excised that may influence patient outcomes, but rather found that a complete regional dissection as opposed to a partial dissection may provide therapeutic benefit ${ }^{9}$. This was further supported in a multi-institutional prospective study comparing 77 non-metastatic patients who underwent RNU with definite template LND compared to 89 who did not undergo LND. The authors found a significantly higher CSS and OS when performing template based LND in patients with renal pelvis tumors pathological stage 2 or higher ${ }^{18}$. Presently, there is no standardized template or algorithm for LND and the extent of the dissection is left to the discretion of the individual surgeon. This issue was identified in our study as significant variation existed in the number of LNs resected by each individual surgeon at the same institution. Recent nodal mapping efforts by Matin et $\mathrm{al}^{19}$, which add to previous work by Kondo et al ${ }^{20}$, thoroughly detailed the predicted landing zones of $\mathrm{LN}$ metastases according to the laterality and location of the tumor. Such efforts aid in the development of anatomical templates that can be implemented both for standardization of surgical dissection and potentially improved oncologic outcomes. A prospective randomized trial evaluating template directed limited versus extended node dissections is needed to be able to sufficiently answer such questions as how many nodes to resect, in what regions and in which patients.

Several important limitations to this study are worth noting. The retrospective nature of this investigation leaves unmeasured confounders difficult to address. Additionally, 31\% of 662 patients did not receive LND for unknown reasons, and the extent of LND and chemotherapy administered were not standardized in this cohort potentially introducing selection bias. We identified several important associations between patient and disease characteristics and number of nodes removed, such that patients who had received preoperative chemotherapy, had higher ASA score and higher pathologic grade were more likely to undergo a more extensive LND. Although, the fact that such variables were associated with higher nodal counts likely suggests a selection bias towards more aggressive dissections in higher risk patients. There was also no central pathology review. However, given the low incidence and heterogeneity of this disease, our series is among the largest single-center studies to date attempting to answer the question of whether or not more LNs removed at the time of RNU results in improved cancer-specific outcomes.

\section{Conclusions}

In the present study we found evidence that a more extensive LND may improve oncologic outcomes in a subset of high-risk patients without significantly increasing OR time or 
serious complications. We described the limitations of evidence and clinical significance of these findings and need for further standardization. Furthermore we have demonstrated that even amongst surgeons from the same institution, substantial heterogeneity exists with regards to the LND performed for patients with UTUC. In sum, we feel that these results have important clinical relevance, which can help continue the movement towards standardization of LND during nephroureterectomy for upper tract tumors.

\section{Acknowledgments}

Funding Source: This research was supported in part by the Sidney Kimmel Center for Prostate and Urologic Cancers. All MSKCC authors were supported by a Cancer Center Support Grant from the National Cancer Institute made to Memorial Sloan Kettering Cancer Center (P30 CA008748). SC is supported by a post-doctoral grant from AFA Insurance. MG is supported by National Institute of Health Ruth L. Kirschstein National Research Service Award T32CA082088.

\section{References}

1. Ozsahin M, et al. Prognostic factors in urothelial renal pelvis and ureter tumours: a multicenter Rare Cancer Network study. European Journal of Cancer. 1999; 35:738-743. [PubMed: 10505034]

2. Zigeuner R, Pummer K. Urothelial carcinoma of the upper urinary tract: surgical approach and prognostic factors. European urology. 2008; 53:720-731. [PubMed: 18207315]

3. Rouprêt M, et al. European guidelines on upper tract urothelial carcinomas: 2013 update. European urology. 2013; 63:1059-1071. [PubMed: 23540953]

4. Secin FP, et al. Evaluation of regional lymph node dissection in patients with upper urinary tract urothelial cancer. International journal of urology. 2007; 14:26-32. [PubMed: 17199856]

5. Rouprêt M, et al. European association of urology guidelines on upper urinary tract urothelial cell carcinoma: 2015 update. European urology. 2015; 68:868-879. [PubMed: 26188393]

6. Kondo T, Tanabe K. Role of lymphadenectomy in the management of urothelial carcinoma of the bladder and the upper urinary tract. International Journal of Urology. 2012; 19:710-721. [PubMed: 22515472]

7. Roscigno M, et al. Lymphadenectomy at the time of nephroureterectomy for upper tract urothelial cancer. European urology. 2011; 60:776-783. [PubMed: 21798659]

8. Brausi MA, et al. Retroperitoneal lymph node dissection (RPLD) in conjunction with nephroureterectomy in the treatment of infiltrative transitional cell carcinoma (TCC) of the upper urinary tract: impact on survival. European urology. 2007; 52:1414-1420. [PubMed: 17507148]

9. Kondo T, et al. Template-based lymphadenectomy in urothelial carcinoma of the upper urinary tract: Impact on patient survival. International journal of urology. 2010; 17:848-854. [PubMed: 20812922]

10. Roscigno M, et al. Prognostic value of lymph node dissection in patients with muscle-invasive transitional cell carcinoma of the upper urinary tract. European urology. 2008; 53:794-802. [PubMed: 18207313]

11. Abe $\mathrm{T}$, et al. Role of lymph node dissection in the treatment of urothelial carcinoma of the upper urinary tract: multi-institutional relapse analysis and immunohistochemical re-evaluation of negative lymph nodes. European Journal of Surgical Oncology (EJSO). 2010; 36:1085-1091. [PubMed: 20832972]

12. Lughezzani G, et al. A critical appraisal of the value of lymph node dissection at nephroureterectomy for upper tract urothelial carcinoma. Urology. 2010; 75:118-124. [PubMed: 19864000]

13. Burger M, et al. No overt influence of lymphadenectomy on cancer-specific survival in organconfined versus locally advanced upper urinary tract urothelial carcinoma undergoing radical nephroureterectomy: a retrospective international, multi-institutional study. World journal of urology. 2011; 29:465-472. [PubMed: 21630120] 
14. Kondo T, et al. Impact of the extent of regional lymphadenectomy on the survival of patients with urothelial carcinoma of the upper urinary tract. The Journal of urology. 2007; 178:1212-1217. [PubMed: 17698147]

15. Roscigno M, et al. The extent of lymphadenectomy seems to be associated with better survival in patients with nonmetastatic upper-tract urothelial carcinoma: how many lymph nodes should be removed? European urology. 2009; 56:512-519. [PubMed: 19559518]

16. Shabsigh A, et al. Defining early morbidity of radical cystectomy for patients with bladder cancer using a standardized reporting methodology. European urology. 2009; 55:164-176. [PubMed: 18675501]

17. Yee A, et al. MP77-14 CAN PRE-OPERATIVE CT IDENTIFY POSITIVE LYMPH NODES IN PATIENTS WITH UPPER TRACT UROTHELIAL CARCINOMA? The Journal of Urology. 2014; 4:e917.

18. Kondo T, et al. Template-based lymphadenectomy in urothelial carcinoma of the renal pelvis: A prospective study. International Journal of Urology. 2014; 21:453-459. [PubMed: 24754341]

19. Matin SF, Sfakianos JP, Espiritu PN, Coleman JA, Spiess PE. Patterns of lymphatic metastases in upper tract urothelial carcinoma and proposed dissection templates. The Journal of urology. 2015; 194:1567-1574. [PubMed: 26094807]

20. Kondo T, et al. Primary site and incidence of lymph node metastases in urothelial carcinoma of upper urinary tract. Urology. 2007; 69:265-269. [PubMed: 17320661] 
Highlights

- LND during radical nephroureterectomy for UTUC is controversial

- $\quad 442$ patients at single institution analyzed from 1976-2015

- $\quad$ LND can be performed safely and without significant increase in OR time

- $\quad$ Subset of high risk patients may benefit 


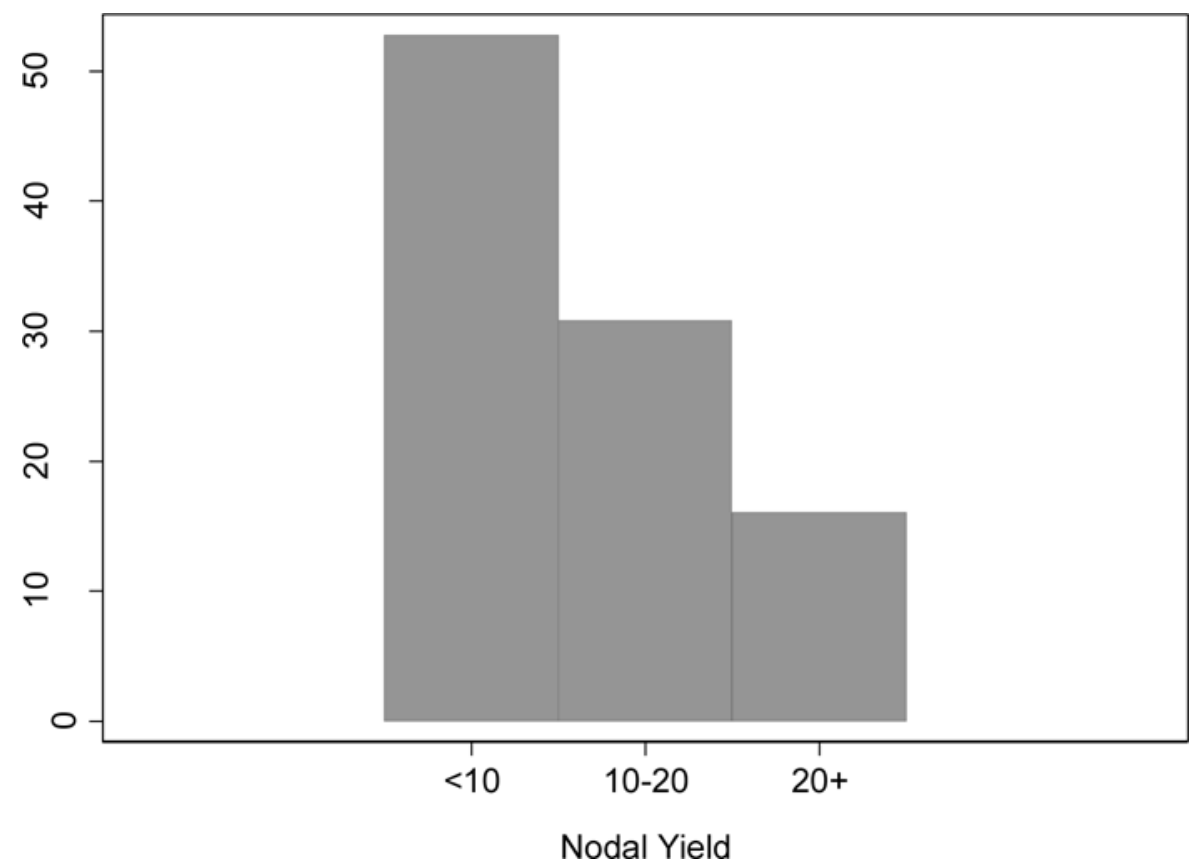

Figure 1.

Distribution of the total number of lymph nodes removed at lymph node dissection. 


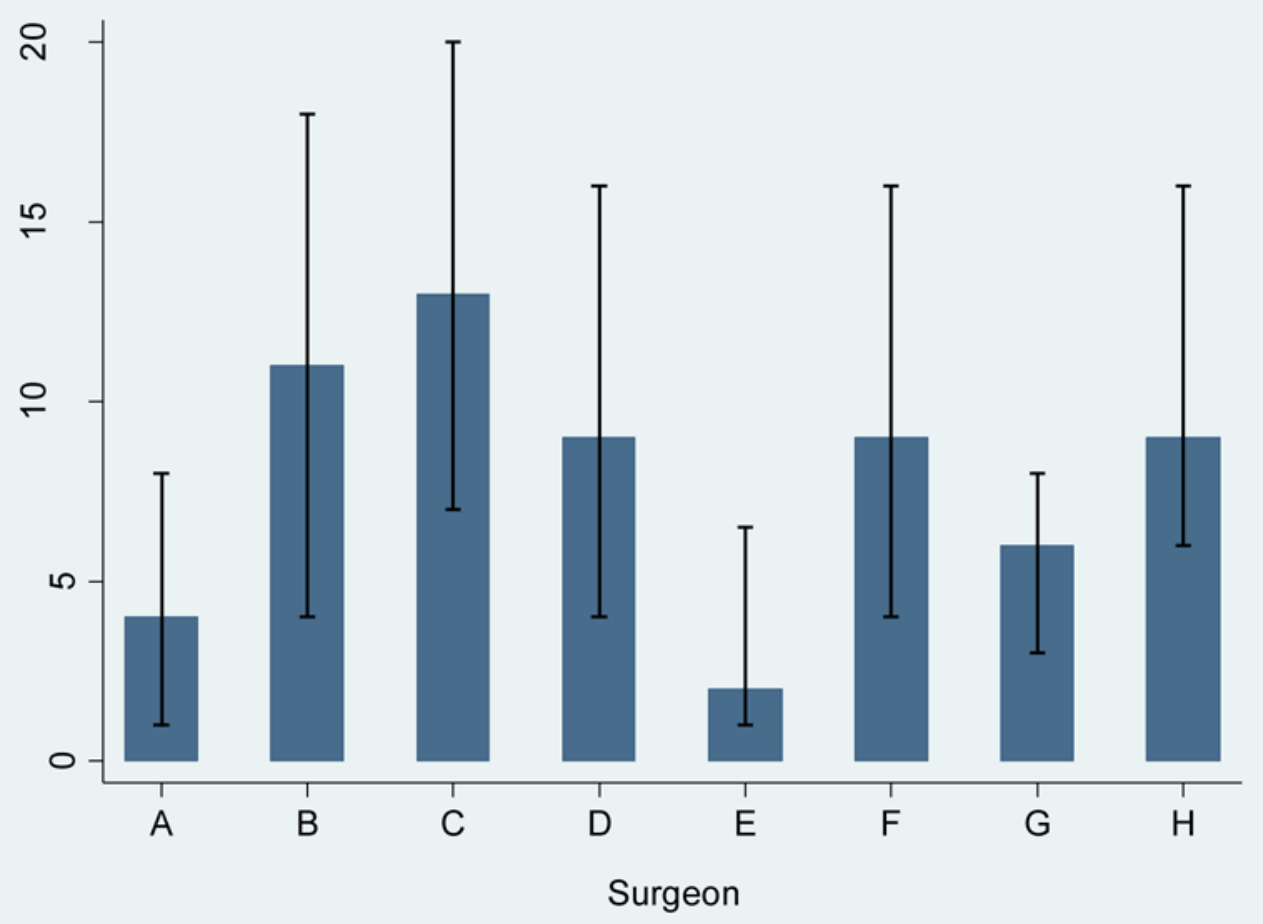

Figure 2.

Distribution of the number of lymph nodes removed by individual surgeon as median and IQR. 


\section{Table 1}

Patient and disease characteristics for patients who had lymph node dissection, $\mathrm{N}=442$. Data are reported as frequency (percent) or median (interquartile range).

\begin{tabular}{|c|c|}
\hline Age & $70(62,76)$ \\
\hline Female & $174(39 \%)$ \\
\hline \multicolumn{2}{|l|}{ Type of surgery } \\
\hline Open Nephroureterectomy & $309(70 \%)$ \\
\hline MIS Nephroureterectomy & $133(30 \%)$ \\
\hline Smoking history & $303(69 \%)$ \\
\hline \multicolumn{2}{|l|}{ ASA score $(\mathrm{N}=424)$} \\
\hline 1 & $4(0.9 \%)$ \\
\hline 2 & $129(30 \%)$ \\
\hline 3 & $278(66 \%)$ \\
\hline 4 & $13(3.1 \%)$ \\
\hline \multicolumn{2}{|l|}{ Pathologic T stage } \\
\hline CIS & $24(5.4 \%)$ \\
\hline T0 & $41(9.3 \%)$ \\
\hline $\mathrm{Ta}$ & $83(19 \%)$ \\
\hline $\mathrm{T} 1$ & $81(18 \%)$ \\
\hline $\mathrm{T} 2$ & $74(17 \%)$ \\
\hline $\mathrm{T} 3$ & $125(28 \%)$ \\
\hline $\mathrm{T} 4$ & $14(3.2 \%)$ \\
\hline High grade $(\mathrm{N}=382)$ & $331(87 \%)$ \\
\hline Multifocal tumor $(\mathrm{N}=373$ ) & $99(27 \%)$ \\
\hline Concomitant CIS & $87(20 \%)$ \\
\hline Pre-operative chemotherapy & $103(23 \%)$ \\
\hline
\end{tabular}


Table 2

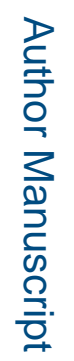

Association between patient and disease characteristics and the number of total nodes removed. Number of nodes reported as median (IQR).

\begin{tabular}{|l|c|c|}
\hline & Total Nodes & p value \\
\hline Smoking history & & 0.6 \\
\hline No & $9(4,16)$ & \\
\hline Yes & $8(3,16)$ & \\
\hline Multifocal tumor & & 0.3 \\
\hline No & $9(4,16)$ & \\
\hline Yes & $8(3,14)$ & \\
\hline Concomitant CIS & & 0.4 \\
\hline No & $9(4,16)$ & \\
\hline Yes & $8(3,14)$ & \\
\hline Pre-operative chemotherapy & & 0.001 \\
\hline No & $8(3,15)$ & \\
\hline Yes & $13(6,18)$ & \\
\hline ASA Score & & 0.071 \\
\hline I/II & $7(3,15)$ & \\
\hline III/IV & $10(4,16)$ & \\
\hline Pathologic Grade & & 0.051 \\
\hline Low & $7(3,12)$ & \\
\hline High & $9(4,16)$ & \\
\hline Pathologic T Stage & & 0.7 \\
\hline CIS & $11(6,16)$ & \\
\hline Ta & $8(3,14)$ & \\
\hline T0 & $13(5,18)$ & \\
\hline T1 & $9(4,16)$ & \\
\hline T2 & & \\
\hline T3 & & \\
\hline T4 & & \\
\hline
\end{tabular}


I

을

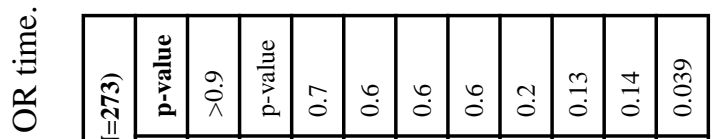

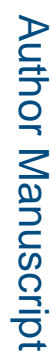

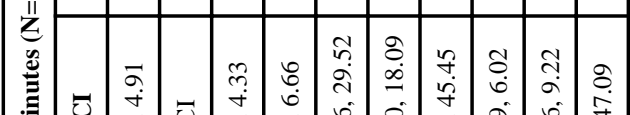

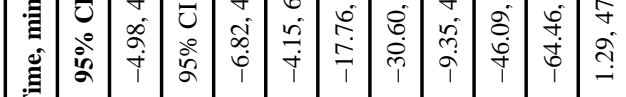

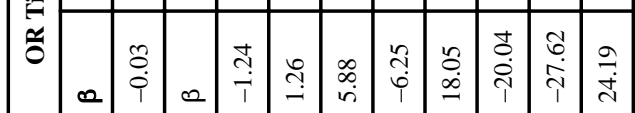

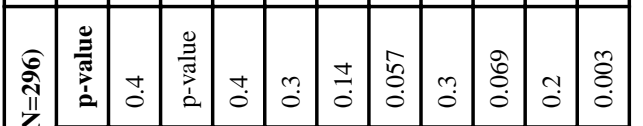

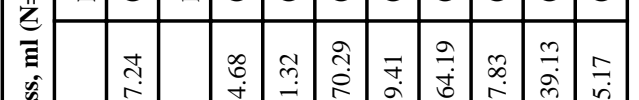

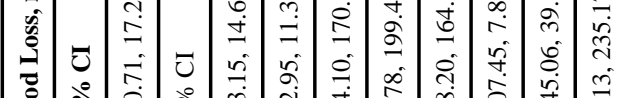

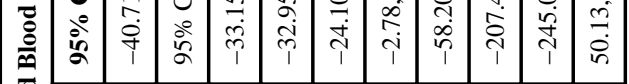

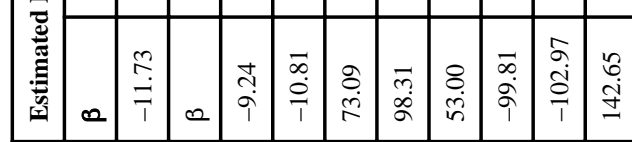

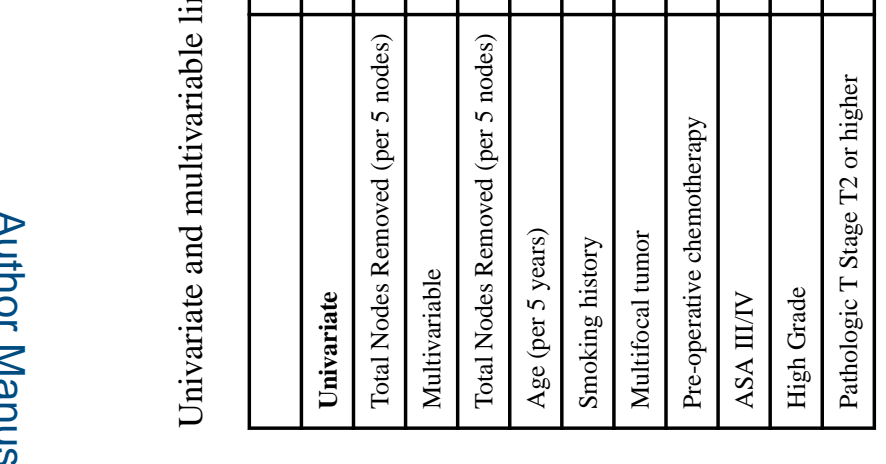

ב্

Urol Oncol. Author manuscript; available in PMC 2018 April 01. 


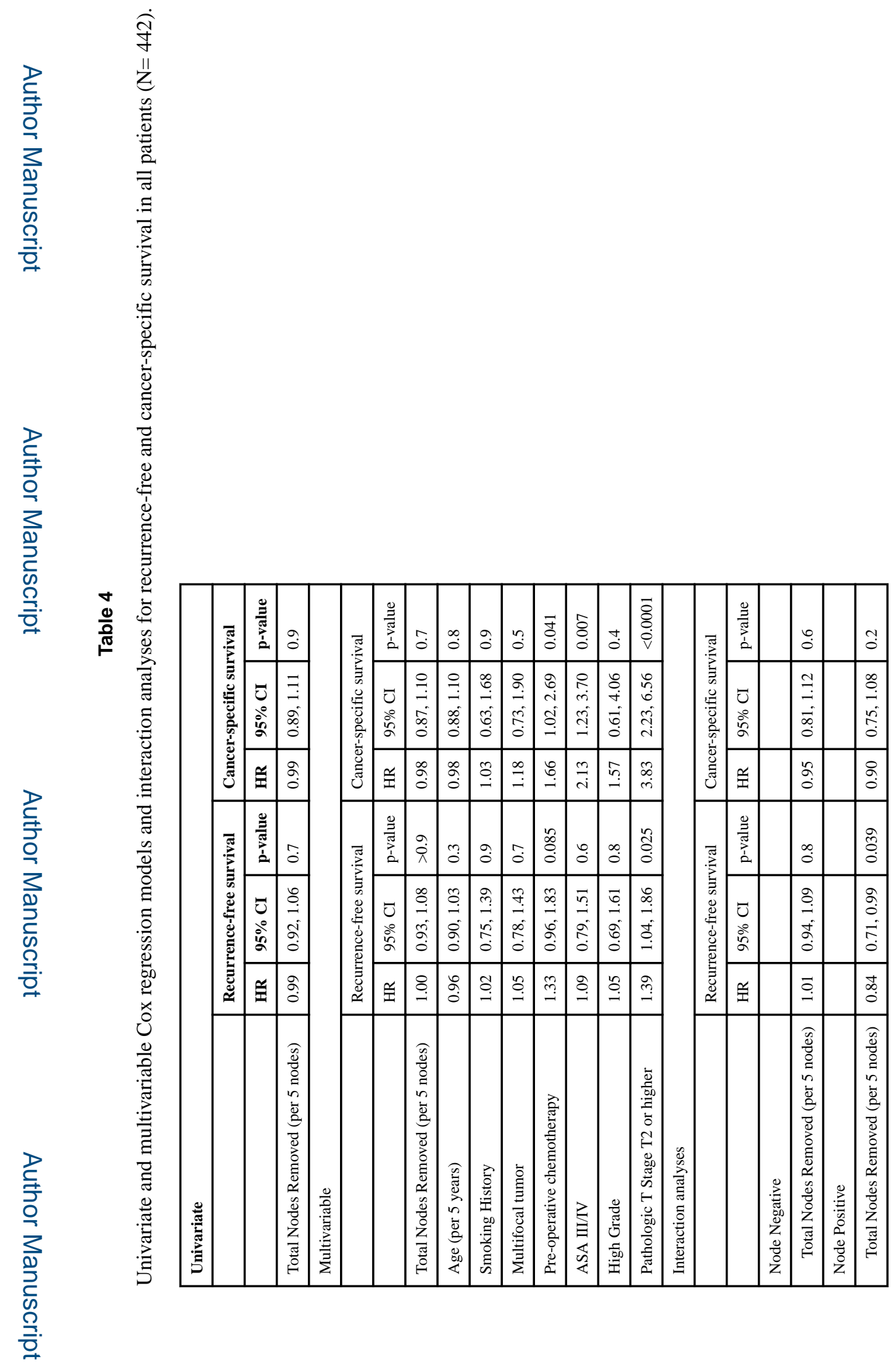

Urol Oncol. Author manuscript; available in PMC 2018 April 01. 


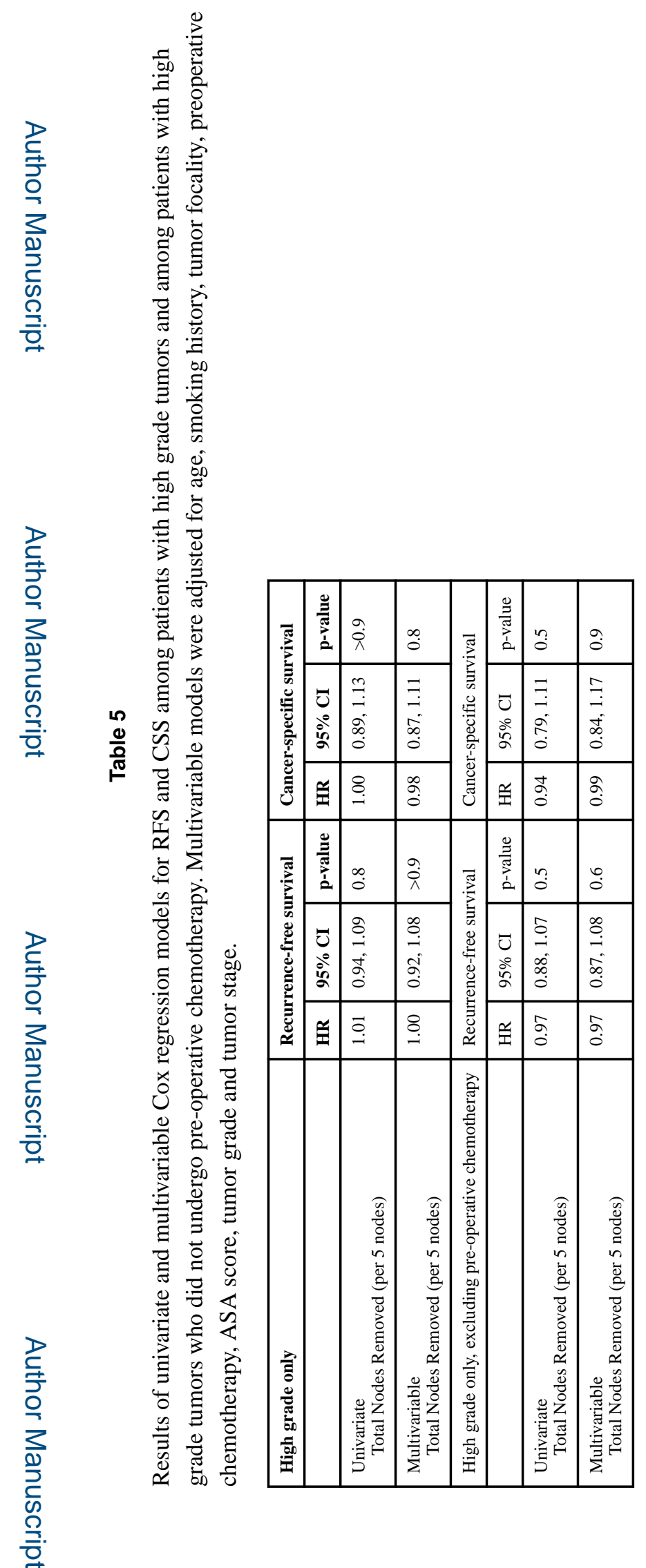

Urol Oncol. Author manuscript; available in PMC 2018 April 01. 
Table 6

Results of univariate Cox regression models for RFS and CSS including interaction terms between number of nodes removed and nodal status.

\begin{tabular}{|l|l|l|l|}
\hline High grade only & \multicolumn{3}{|l|}{ Recurrence-free survival } \\
\hline & HR & $\mathbf{9 5 \%}$ CI & p-value \\
\hline $\begin{array}{l}\text { Node-negative patients } \\
\text { Total Nodes Removed (per 5 nodes) }\end{array}$ & 1.05 & $0.96,1.15$ & 0.3 \\
\hline $\begin{array}{l}\text { Node-positive patients } \\
\text { Total Nodes Removed (per 5 nodes) }\end{array}$ & 0.85 & $0.71,1.02$ & 0.074 \\
\hline High grade only, excluding pre-operative chemotherapy & Recurrence-free survival \\
\hline & HR & $95 \%$ CI & p-value \\
\hline $\begin{array}{l}\text { Node-negative patients } \\
\text { Total Nodes Removed (per 5 nodes) }\end{array}$ & 0.99 & $0.89,1.12$ & 0.9 \\
\hline $\begin{array}{l}\text { Node-positive patients } \\
\text { Total Nodes Removed (per 5 nodes) }\end{array}$ & 0.83 & $0.65,1.06$ & 0.14 \\
\hline Patients treated in 2003 or later & & & \\
\hline & HR & $95 \%$ CI & p-value \\
\hline $\begin{array}{l}\text { Node-negative patients } \\
\text { Total Nodes Removed (per 5 nodes) }\end{array}$ & 1.00 & $0.99,1.02$ & 0.7 \\
\hline $\begin{array}{l}\text { Node-positive patients } \\
\text { Total Nodes Removed (per 5 nodes) }\end{array}$ & 0.96 & $0.93,1.00$ & 0.038 \\
\hline
\end{tabular}

